\title{
Structural Disorder in the Key Lead-Free Piezoelectric Materials, $\mathbf{K}_{x} \mathbf{N a}_{1-x} \mathbf{N b O}_{3}$ and $(1-x) \mathbf{N a}_{0.5} \mathbf{B i}_{0.5} \mathbf{T i O}_{3}+x \mathbf{B a T i O}_{3}$
}

\author{
Jason Schiemer, ${ }^{1,2}$ Ray Withers, ${ }^{1}$ Yun Liu,, ${ }^{1}$ Yiping Guo, ${ }^{1}$ Zhiguo Yi, ${ }^{1}$ and Jian Wang' \\ ${ }^{1}$ Research School of Chemistry, Australian National University, Science Road, Acton, ACT 0200, Australia \\ ${ }^{2}$ Department of Earth Sciences, University of Cambridge, Downing Street, Cambridge CB2 3EQ, UK \\ Correspondence should be addressed to Jason Schiemer; jas263@cam.ac.uk
}

Received 25 October 2013; Accepted 17 November 2013

Academic Editor: Danyang Wang

Copyright (C) 2013 Jason Schiemer et al. This is an open access article distributed under the Creative Commons Attribution License, which permits unrestricted use, distribution, and reproduction in any medium, provided the original work is properly cited.

Using electron diffraction, trends in the local structural behaviour of the $\mathrm{K}_{x} \mathrm{Na}_{1-x} \mathrm{NbO}_{3}(\mathrm{KNN} x)$ and the $(1-x) \mathrm{Na}_{0.5} \mathrm{Bi}_{0.5} \mathrm{TiO}_{3}+$ $x \mathrm{BaTiO}_{3}(\mathrm{NBT}-(x) \mathrm{BT})$ systems are investigated. In $\mathrm{KNN}$, electron diffraction shows a single plane of diffuse intensity perpendicular to [010] across the entire phase diagram, indicating the existence of ferroelectric disorder along this axis. An additional characteristic pattern of diffuse scattering is also observed, involving rods of diffuse intensity running along the $[100]_{\mathrm{p}}{ }^{*}$ and $[001]_{\mathrm{p}}{ }^{*}$ directions of the perovskite substructure and indicative of octahedral tilt disorder about these axes. Similarly, in the NBT- $x$ BT system, rods of diffuse intensity running along the $\langle 100\rangle_{\mathrm{p}}{ }^{*}$ directions of the perovskite substructure are observed, again indicating octahedral tilt disorder. Ferroelectric-like disorder is also observed in highly BT doped samples, and a continuous change from the "rhombohedral" structure of NBT to the "tetragonal" structure of NBT-12BT is seen from characteristic variation in observed superstructure reflections. A crystal chemical rationalisation of these results is performed, and the implications for structure and properties are discussed.

\section{Introduction}

$\mathrm{K}_{x} \mathrm{Na}_{1-x} \mathrm{NbO}_{3}$ (KNN $x$ ) became known as a promising lead-free piezoelectric ceramic system when relatively high piezoelectric coefficients (up to $\sim 300 \mathrm{pC} / \mathrm{N}$ ) along with Curie temperatures of up to $400^{\circ} \mathrm{C}$ were reported by Saito et al. in 2004 [1]. $(1-x) \mathrm{Na}_{0.5} \mathrm{Bi}_{0.5} \mathrm{TiO}_{3}+x \mathrm{BaTiO}_{3}(\mathrm{NBT}-x \mathrm{BT})$ is similarly high performing. Its raw piezoelectric performance is not as high as in $\mathrm{KNN}, \sim 160 \mathrm{pm} / \mathrm{V}$ [2], but it can display huge electrostrictive strains, up to $0.48 \%$ [3], which show promise for electrostrictive actuators with zero strain, as long as they are designed to account for their nonlinear response. These materials are among the few lead-free piezoelectric materials that can compete with lead-based materials in specialized applications. Due to their performance, the structure of materials such as KNN and NBT-BT is of considerable interest in order to establish structure-property relationships and to engineer improvements.

At room temperature in the KNN system, there is long range ferroelectric order in the plane perpendicular to a particular parent perovskite $\langle 001\rangle_{\mathrm{p}}$ direction (usually taken to be the $[010]_{\mathrm{p}}$ direction in $\left.\mathrm{KNN}\right)$. As long range ferroelectric order is established along both the $[100]_{\mathrm{p}}$ and $[001]_{\mathrm{p}}$ directions, the direction of the spontaneous polarization is then along a parent perovskite $\langle 101\rangle_{\mathrm{p}}$ direction, in the case of the end-member $\mathrm{KNbO}_{3}$, and it might be expected to remain largely unchanged across the KNN phase diagram unless there is an unexpectedly strong coupling between ferroelectric ordering and octahedral tilt rotation. There is a pattern of octahedral tilting induced by the introduction of the smaller $\mathrm{Na}^{+}$ions into the perovskite $A$ sites of the originally unrotated, $A m m 2, \mathrm{KNbO}_{3}$ structure type. According to the current phase diagram [4], the onset of $\mathrm{b}^{+}$tilting does not occur until a value of $x=0.4 . b^{+}$tilting is then reported to occur from $x=0.4$ to 0.2 , below which $a^{-}, b^{+}$, and $c^{-}$tilts are reported. The nature of the local structure has not been closely examined, as previous investigations involve powder diffraction using neutrons [5] and X-rays $[4,6]$, which are not very sensitive to short range order.

NBT-BT initially appears simpler than that in KNN. The end member, NBT, is rhombohedral, with ferroelectricity along a particular $[111]_{\mathrm{p}}$ direction. Above $\sim 7 \%$ BT 
doping, the rhombohedral NBT structure transitions to a metrically tetragonal state when observed with powder diffraction methods, while a pseudocubic state exists in the "morphotropic phase boundary" between these two phases. Additionally, there are electric field dependent phase transitions in this system at 7\% BT doping [7], from pseudocubic to metrically tetragonal, which are irreversible.

Very few electron diffraction or diffuse scattering investigations have been performed on these systems. This report seeks to examine disorder present in these materials, in particular, the correlations between the two system and the effects of this disorder on structure determination and properties.

\section{Materials and Methods}

Synthesis of KNN $x$ was performed for samples of composition $x=0.15,0.21,0.35,0.46$, and 0.75 . This was by solid-state reaction for $x=0.15,0.21,0.35$, and 0.75 . Starting reagents were $\mathrm{K}_{2} \mathrm{CO}_{3}, \mathrm{Na}_{2} \mathrm{CO}_{3}$, and $\mathrm{Nb}_{2} \mathrm{O}_{5}$. Mixing and homogenising of reagents were performed in a planetary ball mill, using yttrium stabilised zirconia balls in polymeric tanks. The reagents were ball-milled in stoichiometric ratios for $12 \mathrm{~h}$ under acetone followed by calcining at $900^{\circ} \mathrm{C}$ for $5 \mathrm{~h}$ to drive off carbon dioxide and begin the reaction to form KNN. Ball milling and calcination at $900^{\circ} \mathrm{C}$ were then repeated for homogeneity and complete decomposition. Finally, the powders were ball-milled again for $12 \mathrm{~h}$ and pressed into $20 \mathrm{~mm}$ pellets at $175 \mathrm{MPa}$ in a uniaxial steel press, followed by sintering in a bed of loose powder on Pt foil, in lidded alumina crucibles for $4 \mathrm{~h}$ in the range of $1030^{\circ} \mathrm{C}$ for $\mathrm{KNN}, x=0.15$ to $1110^{\circ} \mathrm{C}$ for $\mathrm{KNN}, x=0.75$. The $x=0.46$ sample was prepared by sol-gel synthesis as described in [8].

NBT- $x$ BT ceramics of composition $x=0,0.04,0.06,0.07$, $0.08,0.10$, and 0.12 were synthesized by solid-state reaction. These samples were from the same batch as described in [9]. Starting reagents were $\mathrm{Na}_{2} \mathrm{CO}_{3}, \mathrm{Bi}_{2} \mathrm{O}_{3}, \mathrm{BaCO}_{3}$, and nano$\mathrm{TiO}_{2}(\sim 20 \mathrm{~nm})$. These were ball-milled for $5 \mathrm{~h}$ in ethanol, followed by calcination at $800^{\circ} \mathrm{C}$ for $2 \mathrm{~h}$. The calcined powder was ball-milled again for $2 \mathrm{~h}$ and dried, followed by the addition of poly(vinylalcohol) binder. This powder was then pressed into pellets with a diameter of $13 \mathrm{~mm}$ in a uniaxial steel press at $200 \mathrm{MPa}$. Finally, these pellets were sintered at $1150^{\circ} \mathrm{C}$ for $2 \mathrm{~h}$ in a covered alumina crucible.

The purity and apparent metric symmetry of the resultant ceramic samples were investigated by X-ray powder diffraction (Siemens D-5000, Cu radiation and Guinier-Hägg camera, $\mathrm{Cu} \mathrm{K}_{\alpha 1}$ radiation), and all samples were found to be pure and free from secondary phases. The Guinier-Hägg patterns collected on the solid-state synthesized KNN samples are shown in Figure 1, below, while the relevant patterns for the KNN 46 sample and the NBT- $x$ BT samples are reported in $[8,9]$ respectively. Electron diffraction patterns (EDPs) were obtained using Philips EM 430 and Philips CM 30 Transmission Electron Microscopes (TEMs) operating at $300 \mathrm{kV}$ on crushed sample powders dispersed onto holey carbon coated, copper grids.

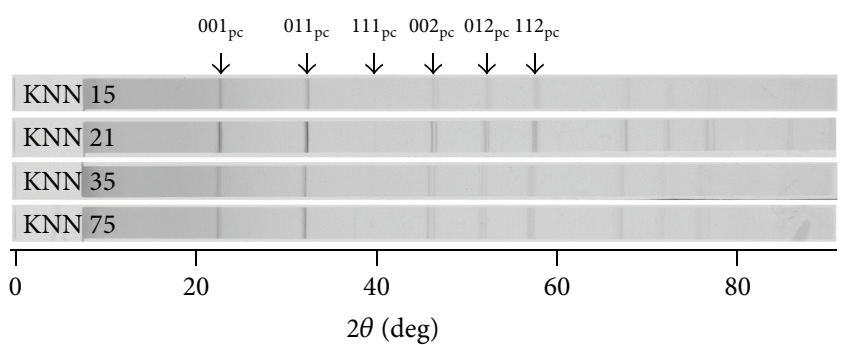

FIGURE 1: Guinier-Hägg X-ray diffraction patterns of solid-state reacted $\mathrm{KNN}$, showing only the expected perovskite-related peaks. The subscript "pc" indicates pseudocubic indexing.

\section{Results and Discussion}

3.1. KNN Average Structure. In all discussions on EDPs, a subscript $\mathrm{p}$ denotes that the indexing is with respect to a parent perovskite subcell. EDPs of KNN as a function of composition are shown in Figures 2(a) and 2(b). We start with the unrotated, high $x$ samples and then consider the octahedrally rotated phases that occur when $x$ is reduced. With $x=0.75$ (see Figure 2(a)), there are no additional superstructure reflections, and the structure appears to be Amm2, as is well known for $\mathrm{KNbO}_{3}$. There are, however, faint diffuse features, which will be discussed subsequently. From $x=0.46$ down to $x=0.21$, a $G_{\mathrm{p}} \pm 1 / 2(101)_{\mathrm{p}}$ type spot occurs, with the intensity of this spot increasing with decreasing potassium content. A sharp $G_{\mathrm{p}} \pm 1 / 2(111)_{\mathrm{p}}$ spot appears in $x=0.15$ (Figure $2(\mathrm{~b})$ ) in addition to $G_{\mathrm{p}} \pm 1 / 2(101)_{\mathrm{p}}$. A diffuse feature exists at $G_{\mathrm{p}} \pm 1 / 2(111)_{\mathrm{p}}$ in all patterns from $x=0.46$ to $x=0.21$. Weak spots associated with a doubled $b$ axis can be seen in the $x=0.15$.

3.2. NBT-BT Average Structure. EDPs of NBT-BT as a function of BT dopant are shown in Figures 2(a) and 2(b). We start with the "rhombohedral" NBT end member and examine the superstructure reflections as BT is introduced. Previous refinements suggest that the structure remains rhombohedral with $x<0.06$, going pseudocubic at $0.06<0.07$ and tetragonal for $x>0.07$. Examination of the diffraction patterns does not reproduce these findings. There is, in fact, a continuous change of intensity between the "rhombohedral" $G_{\mathrm{p}} \pm 1 / 2(111)_{\mathrm{p}}$ spot and the "tetragonal" $G_{\mathrm{p}} \pm 1 / 2(101)_{\mathrm{p}}$ spot, both of which exist to some degree, even in pure NBT. Increasing the BT content reduces the intensity of the "rhombohedral" spot and increases the intensity of the "tetragonal" spot, but neither disappears completely. The width of the "tetragonal" peak is also noticeably higher than the parent reflections, even in NBT-12BT.

3.3. Diffuse Scattering. In all KNN EDPs, a single plane of diffuse scattering is observed perpendicular to [010], as expected from being in the singly disordered ferroelectric (orthorhombic) state [11] (chevron-headed arrows with dashed tails in Figures 2(a) and 2(b)). This diffuse scattering indicates ordered ferroelectricity in the 101 plane, 


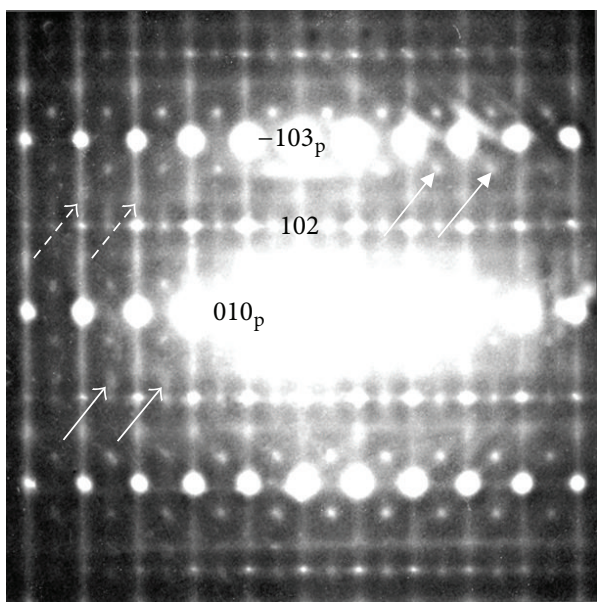

(a)

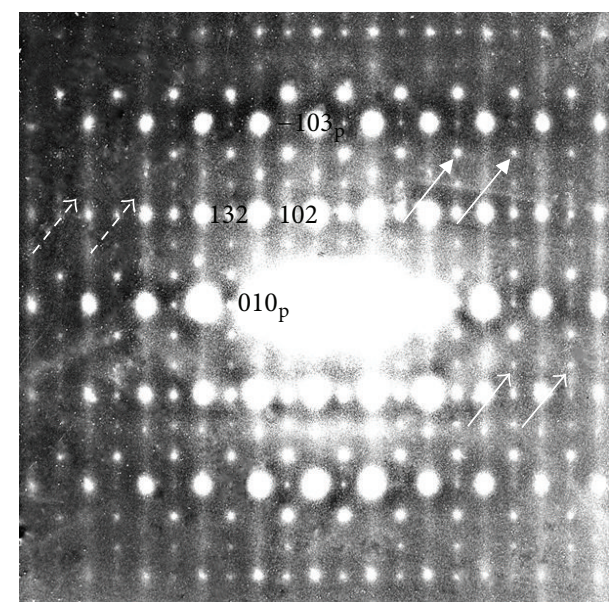

(b)

FIGURE 2: Electron diffraction patterns along a $-3,0,-1_{\mathrm{p}}$ axis in KNN $x$ with (a) $x=0.75$ and (b) $x=0.15$.

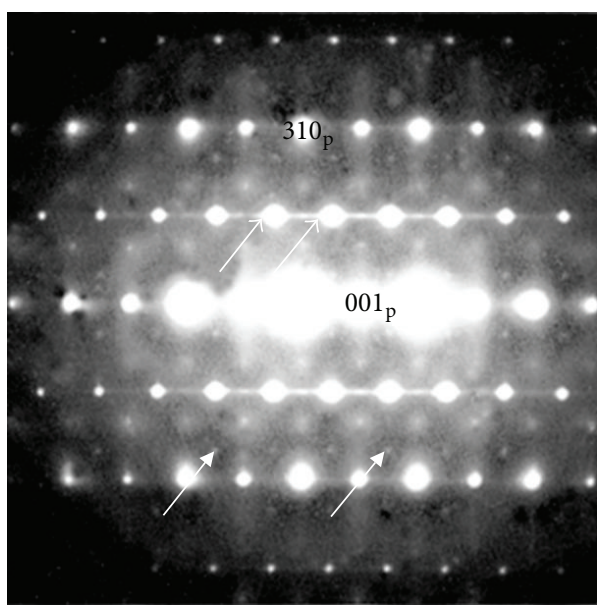

(a)

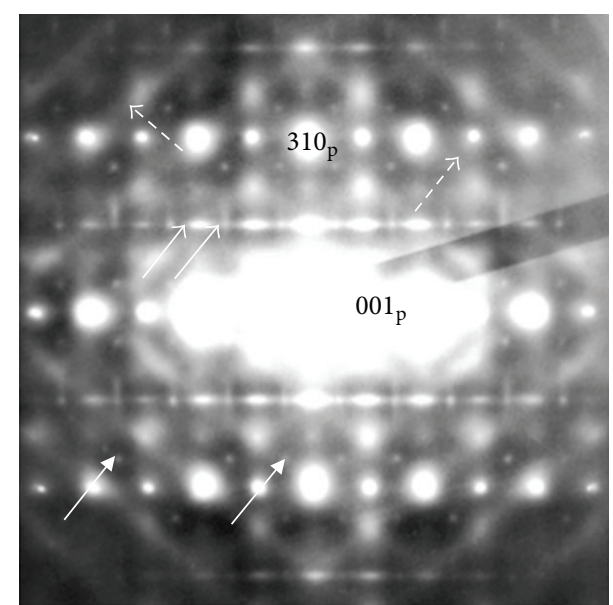

(b)

FIGURE 3: Electron diffraction patterns along a $301_{\mathrm{p}}$ axis in NBT- $x$ BT with (a) $x=0$ and (b) $x=0.12$.

with columnar disorder along [010]. In NBT, no ferroelectric diffuse is seen, again, as expected from being the fully ordered ferroelectric (rhombohedral) state. However, examination of the diffraction patterns from NBT-12BT (Figure 3(b)) shows planes of diffuse intensity perpendicular to $[111]_{\mathrm{p}}$, indicating ferroelectric disorder along these axes (chevron-headed arrows with dashed tails), generated by the addition of BT. Additionally, $G_{\mathrm{p}} \pm[1 / 2,1 / 2, \xi]_{\mathrm{p}}{ }^{*}$ type streaks of diffuse intensity, denoted by chevron-headed arrows with solid tails, are observed from $1 / 2(111)_{p}$ to $1 / 2(110)_{\mathrm{p}}$ and to $1 / 2(011)_{\mathrm{p}}$ at all compositions in $\mathrm{KNN}$ and from $1 / 2(111)_{\mathrm{p}}$ to $1 / 2(110)_{\mathrm{p}}$, to $1 / 2(101)_{\mathrm{p}}$, and to $1 / 2(011)_{p}$ in NBT-BT. These one-dimensional rods of diffuse appear both in the plane and as diffuse "spots" (solidheaded arrows with solid tails) which arise from the Ewald sphere cutting through one of the rods of diffuse intensity. In $\mathrm{KNN}$, these can be indexed as $G_{\mathrm{p}} \pm[\xi, 1 / 2,1 / 2]_{\mathrm{p}}{ }^{*}$ or $G_{\mathrm{p}} \pm[1 / 2,1 / 2, \xi]_{\mathrm{p}}{ }^{*}$ and in NBT-BT as $G_{\mathrm{p}} \pm[\xi, 1 / 2,1 / 2]_{\mathrm{p}}{ }^{*}$ or $G_{\mathrm{p}} \pm[1 / 2,1 / 2, \xi]_{\mathrm{p}}{ }^{*}$ or $G_{\mathrm{p}} \pm[1 / 2, \xi, 1 / 2]_{\mathrm{p}}{ }^{*}$. This effect also gives rise to apparent "spots" of the type $G_{\mathrm{p}} \pm[1 / 2,1 / 2,0]_{\mathrm{p}}{ }^{*}$ and $G_{\mathrm{p}} \pm[1 / 2,1 / 2,1 / 2]_{\mathrm{p}}{ }^{*}$, which can be misleading in patterns which show no other evidence that the "reflections" originate from diffuse rods. In NBT-BT, the continuous movement of intensity between the "rhombohedral" $G_{p} \pm$ $1 / 2(111)_{p}$ spot and the "tetragonal" $G_{p} \pm 1 / 2(101)_{p}$ spot is accompanied by a continuous diffuse streak between these spots for all compositions and the $G_{\mathrm{p}} \pm 1 / 2(101)_{\mathrm{p}}$ spot remains diffuse and anisotropic, even when relatively strong. Denoyer et al. [12] observed streaking of the type $G_{\mathrm{p}} \pm[\xi, 1 / 2,1 / 2]_{\mathrm{p}}{ }^{*}$ in $\mathrm{NaNbO}_{3}$ near a phase transition at $641 \mathrm{~K}$ and assigned it to oxygens in the structure. Indeed, the directions and locations of these streaks in both KNN and NBT-BT suggest octahedral tilt twin disorder along their axes, as does the absence of even harmonics of the type $G_{\mathrm{p}} \pm[1,1,2 \xi]_{\mathrm{p}}{ }^{*} / G_{\mathrm{p}} \pm[2 \xi, 1,1]_{\mathrm{p}}{ }^{*}$.

In $\mathrm{KNN}$, the diffuse rods are clearer for lower sodium compositions. This is unsurprising, as $\mathrm{KNbO}_{3}$ is untilted, 


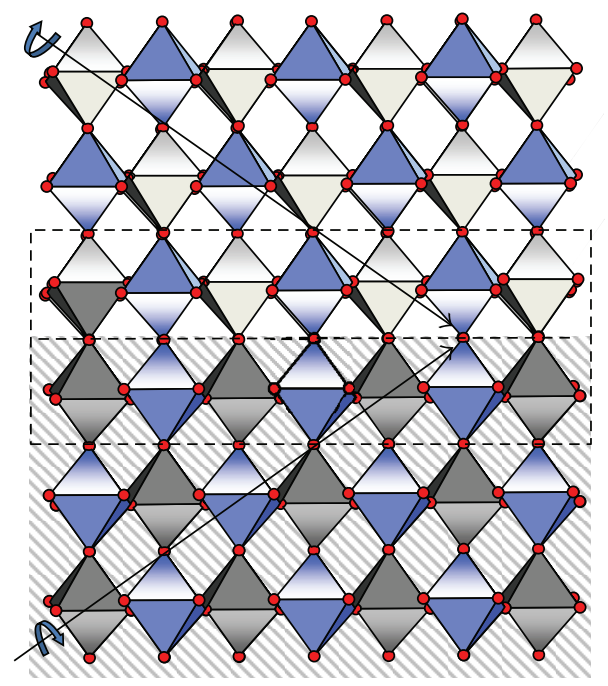

FIgURE 4: Tilt twin region in NBT/NBT-BT showing two rhombohedral twins with different polarization directions meeting in a tetragonal tilt twin region, as shown by the dashed box. Modified version reproduced with permission from [10].

while $\mathrm{NaNbO}_{3}$ is highly tilted, and is due to the fact that tilts increase $A$ site valence and decrease $B$ site valence, and sodium is much smaller than potassium. Disorder in the tilts gives them zero amplitude in refinements of the average structure. The continuous nature of the changes of tilt in KNN implies that there are no phase boundaries with ferroelectric polarization rotation, which are known to lead to temperature independent phase boundaries with enhanced properties. Similarly in NBT, tilt disorder is likely to stem from the significant ionic site size requirement mismatch between a $\mathrm{Bi}^{\mathrm{III}}$ and $\mathrm{Na}^{\mathrm{I}}$ ion. Performing bond valence sums on the ICSD structure, the valence for $\mathrm{Na}$ is found to be $0.12 \mathrm{v}$.u. high and $\mathrm{Bi}$ is found to be $0.60 \mathrm{v}$.u. low. Ordering of these ions may be expected, but this is likely to be frustrated by kinetic and ferroelectric requirements, and no superstructure is observed. Tilt twin disorder of the kind shown in Figure 4 may be occurring, with the larger twin boundary sites more likely to contain the sodium ion. The final question in NBT$\mathrm{BT}$ is with regard to what occurs when BT is added to NBT, which is effectively the addition of $\mathrm{Ba}^{\mathrm{II}}$ to the structure, which requires a much larger ionic cavity than $\mathrm{Na}^{\mathrm{I}}$ or $\mathrm{Bi}^{\mathrm{III}}$. This effect is likely to force the $\mathrm{Ba}$ ions to occupy twin boundary regions, and it appears that the twin boundary region is able to expand to a multiple unit cell thickness, due to the driving force of accommodating $\mathrm{Ba}$, creating layers of tetragonal BT-like material between twins of rhombohedral NBT-like material.

If the dopant concentration is sufficiently high, then this twin boundary region will become the "bulk" and the rhombohedral "bulk" region will become the twin boundary, giving a continuous intensity shift from rhombohedral-like reflections to tetragonal-like reflections, as is experimentally observed. This has been observed directly in NBT-6BT with $3 \% \mathrm{KNN}$ [13], with polar nanoregions of $R 3 \mathrm{c}$ and $\mathrm{P} 4 \mathrm{bm}$ structure reported. This mixing of structures induces disorder in the ferroelectric direction observed from diffuse scattering and may lead to the reported antiferroelectric behaviour and giant strain in NBT-BT.

\section{Conclusions}

In this report we have examined the local structure and disorder in NBT-BT and KNN. This investigation has shown a single, undisturbed ferroelectric plane of diffuse scattering in all compositions of KNN. Additionally, KNN shows disordered octahedral tilting at all compositions due to the mismatch in size between $\mathrm{K}$ and $\mathrm{Na}$ atoms. This disordered tilting behaviour locks into some order at the composition dependent tilt transitions, but no polarisation rotation occurs.

NBT-BT is shown to also have octahedral tilt disorder at all compositions, but ferroelectric disorder only occurs with BT doping. Continuous transfer of intensity from the "rhombohedral" $G_{p} \pm 1 / 2(111)_{p}$ spot to the "tetragonal" $G_{p} \pm$ $1 / 2(101)_{p}$ spot with increasing BT content is explained as an expansion of the stacking fault boundary regions in the material due to the ionic size of $\mathrm{Ba}$. This explanation also gives a reason for observed ferroelectric disorder in NBT12BT and may supply information about the origins of high electrostrictive response and antiferroelectric behaviour in NBT-BT.

\section{References}

[1] Y. Saito, H. Takao, T. Tani et al., "Lead-free piezoceramics," Nature, vol. 432, no. 7013, pp. 84-87, 2004.

[2] D. Maurya, C. W. Ahn, and S. Priya, "Structural and electrical characterization of lead-free $(1-x)\left(\mathrm{Na}_{1 / 2} \mathrm{~B}_{1 / 2}\right) \mathrm{TiO}_{3-x} \mathrm{BaTiO}_{3}$ piezoelectric ceramics," in Advances in Electroceramic Materials II: Ceramic Transactions, pp. 47-54, Wiley, 2010.

[3] Y. Guo, M. Gu, H. Luo, Y. Liu, and R. L. Withers, "Compositioninduced antiferroelectric phase and giant strain in lead-free $\left(\mathrm{Na}_{y}, \mathrm{Bi}_{z}\right) \mathrm{Ti}_{1-x} \mathrm{O}_{3(1-x)}-x \mathrm{BaTiO}_{3}$ ceramics," Physical Review B, vol. 83, Article ID 054118, 7 pages, 2011.

[4] D. W. Baker, P. A. Thomas, N. Zhang, and A. M. Glazer, "A comprehensive study of the phase diagram of $\mathrm{K}_{x} \mathrm{Na}_{1-x} \mathrm{NbO}_{3}$," Applied Physics Letters, vol. 95, no. 9, Article ID 091903, 3 pages, 2009.

[5] M. Ahtee and A. W. Hewat, "Structural phase transitions in sodium-potassium niobate solid solutions by neutron powder diffraction," Acta Crystallographica A, vol. 34, pp. 309-317, 1978.

[6] M. Ahtee and A. M. Glazer, "Lattice parameters and tilted octahedra in sodium-potassium niobate solid solutions," Acta Crystallographica A, vol. 32, pp. 434-446, 1976.

[7] J. E. Daniels, W. Jo, J. Rodel, and J. L. Jones, "Electric-fieldinduced phase transformation at a lead-free morphotropic phase boundary: case study in a $93 \%\left(\mathrm{Bi}_{0.5} \mathrm{Na}_{0.5}\right) \mathrm{TiO}_{3} 7 \%$ $\mathrm{BaTiO}_{3}$ piezoelectric ceramic," Applied Physics Letters, vol. 95, Article ID 032904, 3 pages, 2009.

[8] Z. Yi, Y. Liu, M. A. Carpenter, J. Schiemer, and R. L. Withers, " $\mathrm{K}_{0.46} \mathrm{Na}_{0.54} \mathrm{NbO}_{3}$ ferroelectric ceramics: chemical synthesis, electro-mechanical characteristics, local crystal chemistry and elastic anomalies," Dalton Transactions, vol. 40, no. 18, pp. $5066-$ 5072, 2011.

[9] Y. Guo, Y. Liu, R. L. Withers, F. Brink, and H. Chen, "Large electric field-induced strain and antiferroelectric behavior in 
$(1-x)\left(\mathrm{Na}_{0.5} \mathrm{Bi}_{0.5}\right) \mathrm{TiO}_{3-x} \mathrm{BaTiO}_{3}$ ceramics," Chemistry of Materials, vol. 23, no. 2, pp. 219-228, 2011.

[10] Y. Liu, L. Norén, A. J. Studer et al., "Response of intergrown microstructure to an electric field and its consequences in the lead-free piezoelectric bismuth sodium titanate," Journal of Solid State Chemistry, vol. 187, pp. 309-315, 2012.

[11] R. Comes, M. Lambert, and A. Guinier, "Désordre linéaire dans les cristaux (cas du silicium, du quartz, et des pérovskites ferroélectriques)," Acta Crystallographica A, vol. 26, pp. 244254, 1970.

[12] F. Denoyer, R. Comes, and M. Lambert, "X-ray diffuse scattering from $\mathrm{NaNbO}_{3}$ as a function of temperature," Acta Crystallographica A, vol. 27, pp. 414-420, 1971.

[13] L. A. Schmitt and H. Kleebe, "Single grains hosting two space groups: a transmission electron microscopy study of a lead-free ferroelectric," Functional Materials Letters, vol. 3, no. 1, pp. 55$58,2010$. 

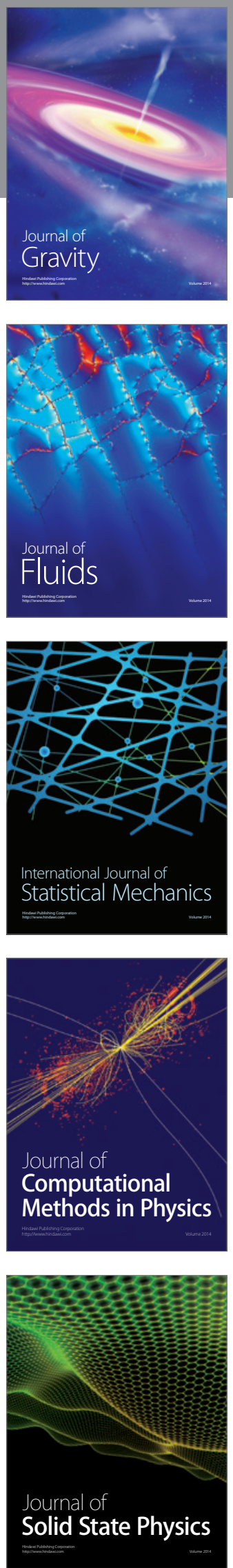

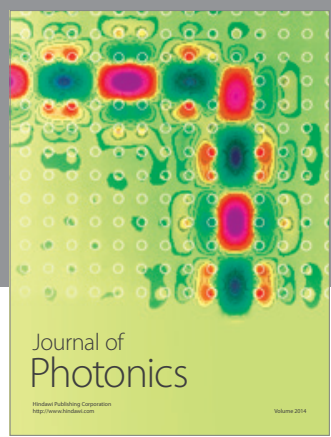

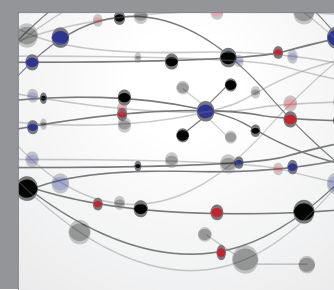

The Scientific World Journal

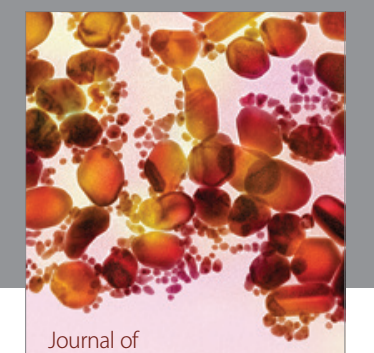

Soft Matter
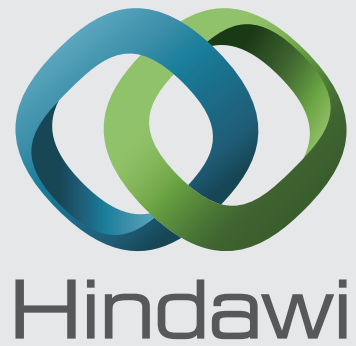

Submit your manuscripts at

http://www.hindawi.com
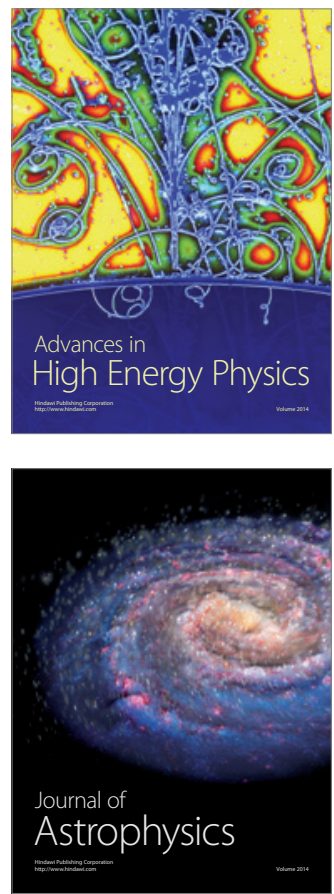
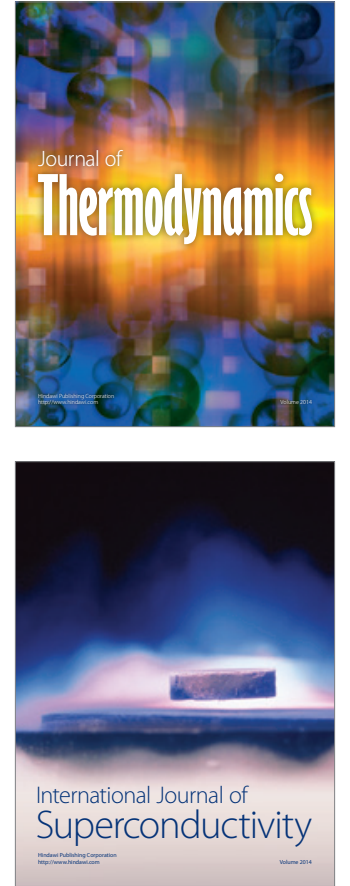
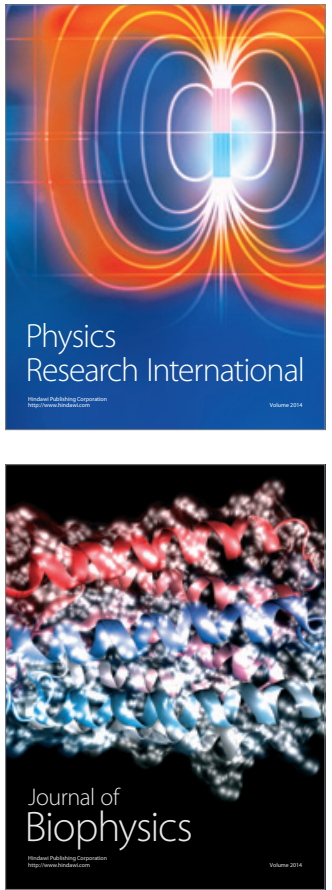
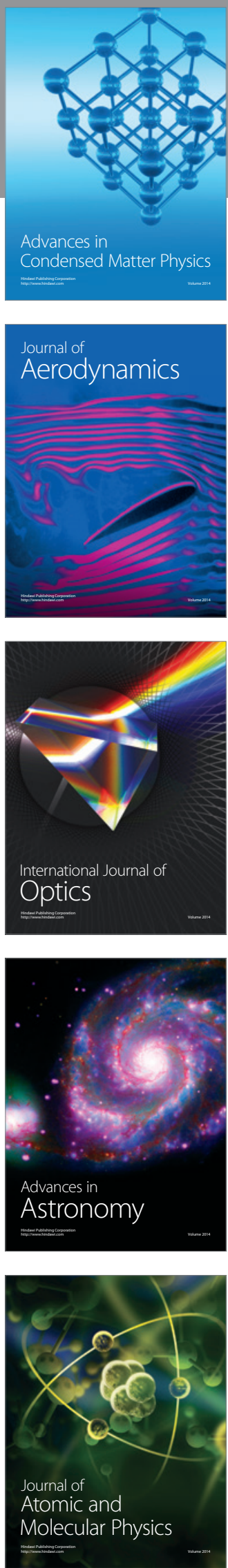